\title{
Tobacco Smoking in People Is Not Associated with Altered 18-kDa Translocator Protein Levels: A PET Study
}

\author{
Ansel T. Hillmer ${ }^{1-4}$, David Matuskey ${ }^{1-3,5}$, Yiyun Huang ${ }^{1,3}$, Nabeel Nabulsi ${ }^{1,3}$, Jim Ropchan ${ }^{1,3}$, Richard E. Carson ${ }^{1-4}$, \\ Stephanie S. O'Malley², and Kelly P. Cosgrove ${ }^{1-3,6}$ \\ ${ }^{1}$ Department of Radiology and Biomedical Imaging, Yale University School of Medicine, New Haven, Connecticut; ${ }^{2}$ Department of \\ Psychiatry, Yale University School of Medicine, New Haven, Connecticut; ${ }^{3}$ Yale University PET Center, Yale University School of \\ Medicine, New Haven, Connecticut; ${ }^{4}$ Department of Biomedical Engineering, Yale School of Engineering and Applied Science, New \\ Haven, Connecticut; ${ }^{5}$ Department of Neurology, Yale University School of Medicine, New Haven, Connecticut; and ${ }^{6}$ Department of \\ Neuroscience, Yale University School of Medicine, New Haven, Connecticut
}

The effects of tobacco smoking on the immune system of the brain are not well elucidated. Although nicotine is immunosuppressive, other constituents in tobacco smoke have inflammatory effects. PET imaging of the 18-kDa translocator protein (TSPO) provides a biomarker for microglia, the primary immunocompetent cells of the brain. This work compared brain TSPO levels in 20 tobacco smokers (abstinent for at least $2 \mathrm{~h}$ ) and 20 nonsmokers using a fully quantitative modeling approach for the first time, to our knowledge. Methods: ${ }^{11} \mathrm{C}-\mathrm{PBR} 28\left(\mathrm{~N}-\left(\left(2-\left(\operatorname{methoxy}-{ }^{11} \mathrm{C}\right)-\right.\right.\right.$ phenyl) methyl)- $N$-(6-phenoxy-3-pyridinyl)acetamide) PET scans were acquired with arterial blood sampling to estimate the metabolite-corrected input function. ${ }^{11} \mathrm{C}-\mathrm{PBR} 28$ volumes of distribution were estimated throughout the brain with multilinear analysis. Results: Statistical analyses revealed no evidence of significant differences in regional ${ }^{11} \mathrm{C}$-PBR28 volumes of distribution between smokers and nonsmokers (whole-brain Cohen $d=0.09$ ) despite adequate power to detect medium effect sizes. Conclusion: These findings inform previous PET studies reporting lower TSPO radiotracer concentrations in the brain (measured as SUV) for tobacco smokers than for nonsmokers by demonstrating the importance of accounting for radiotracer concentrations in plasma. These findings suggest that nonsmokers and smokers have comparable TSPO levels in the brain. Additional work with other biomarkers is needed to fully characterize the effects of tobacco smoking on the brain immune system.

Key Words: PET imaging; TSPO; tobacco smoking

J Nucl Med 2020; 61:1200-1204

DOI: 10.2967/jnumed.119.237735

$\mathbf{T}$ obacco smoking is a leading cause of preventable disease and death, annually responsible for 6 million deaths (1). Many negative health consequences associated with tobacco smoking, including cancer, respiratory infection, and bacterial meningitis (2), involve altered immune function. These immune impairments may be mediated by free radicals, toxic aldehydes, or other

Received Oct. 4, 2019; revision accepted Dec. 19, 2019.

For correspondence or reprints contact: Ansel T. Hillmer, Yale University School of Medicine, 2 Church St. S., \#314, New Haven, CT 06511.

E-mail: ansel.hillmer@yale.edu

Published online Jan. 31, 2020.

COPYRIGHT (C 2020 by the Society of Nuclear Medicine and Molecular Imaging. inflammatory constituents present in tobacco smoke (3). Tobacco smokers exhibit significantly higher circulatory levels of the proinflammatory proteins C-reactive protein and interleukin-6 $(4,5)$ than nonsmokers, potentially contributing to an increased prevalence of diseases characterized by chronic inflammation (6).

Although the effects of tobacco smoking on peripheral immune signaling have yet to be fully elucidated, even less is known about the effects on the immune system of the brain. Knowledge of these effects is important because dysregulated brain immune signaling contributes to compulsive drug use and common comorbidities of drug use $(7,8)$. Microglia, the primary immunocompetent cells of the brain, are critical to maintaining neuronal homeostasis and synaptic plasticity (9). Consequently, neuroimmune pharmacotherapies, such as minocycline and ibudilast, have generated interest for smoking cessation (10). However, tobacco smoking has complex effects on the brain. Although many constituents of tobacco smoke are proinflammatory, nicotine itself has antiinflammatory effects (11). Additionally, the blood-brain barrier likely alters smoke-constituent exposure in the brain relative to the body. Therefore, research directly examining neuroimmune markers in tobacco smokers is critical to inform approaches that might improve health outcomes.

An imaging approach to studying the immune system of the brain is PET imaging of the $18-\mathrm{kDa}$ translocator protein (TSPO) (12). TSPO is expressed primarily in microglia and to a lesser extent in astrocytes and endothelial cells. The activation of microglia in response to pathogens or injury (13) increases TSPO expression in microglia. Microglial activation is a hallmark of the brain immune response and is critical for repairing cells and reestablishing homeostasis $(14,15)$. PET radioligands with high affinity and specificity for TSPO, such as ${ }^{11} \mathrm{C}-\mathrm{PBR} 28\left(N\right.$-( (2-(methoxy- $\left.{ }^{11} \mathrm{C}\right)$-phenyl)methyl)$N$-(6-phenoxy-3-pyridinyl)acetamide) (16-18), can be used to estimate the ligand distribution volume $\left(V_{\mathrm{T}}\right)(19)$, which is proportional to the number of in vivo sites available for ${ }^{11} \mathrm{C}$-PBR2 8 binding (i.e., TSPO availability). The goal of this work was to compare TSPO levels in tobacco smokers and nonsmokers using fully quantitative PET imaging. On the basis of preclinical research showing that exposure to tobacco smoke increased neuroimmune markers $(20,21)$, we hypothesized that tobacco smokers would have higher TSPO levels than nonsmokers. Interestingly, 2 previous studies reported significantly decreased brain concentrations of a TSPOspecific radioligand in the brains of tobacco smokers compared with nonsmokers $(22,23)$. A major limitation of these reports was the outcome measure of normalized radioligand concentrations in 
the brain (SUV). For all PET radiotracers, brain SUVs alone do not account for radioligand concentrations in plasma. Because the plasma input function is not known, brain SUV may be dissociated from target protein levels (such as TSPO). Thus, a secondary goal of this work was to compare fully quantitative $V_{\mathrm{T}}$ measures of TSPO availability with semiquantitative SUV measures.

\section{MATERIALS AND METHODS}

\section{Subjects}

Twenty tobacco smokers (15 men and 5 women) and 20 agematched healthy nonsmokers (13 men and 7 women) underwent an ${ }^{11}$ C-PBR28 PET scan with arterial blood sampling and an anatomic MRI scan. Before scanning, the subjects were genotyped for the rs6971 single-nucleotide polymorphism, which affects ${ }^{11} \mathrm{C}-\mathrm{PBR} 28$ affinity for the TSPO site (24), as previously described (25). T/T homozygotes (low-affinity binders) were excluded. Data from a subset of subjects (4 smokers and 10 nonsmokers) have been reported previously $(26,27)$.

All procedures were approved by the Yale School of Medicine Human Investigation Committee and the Radiation Safety Committee. Written informed consent, approved by the Human Investigation Committee, was obtained from all subjects before participation in this study. All subjects underwent screening procedures that included a physical examination, electrocardiogram, routine blood tests, and urine toxicologic analysis. None had any significant findings on screening or any history of major medical disorders, and none met any of the criteria for current or past psychiatric or substance abuse diagnosis (other than nicotine dependence for cigarette smokers) as described in the 4th edition of the Diagnostic and Statistical Manual of Mental Disorders. All female participants were required to have a negative pregnancy test on the scanning day before receiving the radiotracer. Smokers were required to have smoked cigarettes daily for at least $1 \mathrm{y}$. A current smoking status was confirmed during intake by carbon monoxide levels greater than 10 parts per million (ppm) and urine cotinine levels greater than $150 \mathrm{ng} / \mathrm{mL}$.

On the scanning day, the subjects completed a questionnaire characterizing mood, including the Beck Depression Inventory (28). Tobacco smokers completed measures of tobacco smoking behavior, including the Fagerström Test for Nicotine Dependence (29), the Minnesota Nicotine Withdrawal Scale (30), and the Urge to Smoke Questionnaire (31). Tobacco smokers were abstinent for a minimum of $2 \mathrm{~h}$ before scanning and a maximum of $14 \mathrm{~h}$. Levels of carbon monoxide and urine cotinine, the primary metabolite of nicotine, were also collected on the scanning day.

\section{Data Acquisition}

${ }^{11} \mathrm{C}-\mathrm{PBR} 28$ was produced as previously described (25). PET imaging data were acquired with a High-Resolution Research Tomograph (CTI/Siemens Medical Solutions). Head motion data were acquired with an optical motion-tracking device (Vicra; NDI Systems). PET imaging scans began with acquisition of a ${ }^{137} \mathrm{Cs}$ transmission scan over $6 \mathrm{~min}$. Emission data acquisition began simultaneously with bolus injection of $540 \pm 181 \mathrm{MBq}$ of ${ }^{11} \mathrm{C}$-PBR28 and continued for $120 \mathrm{~min}$. Arterial blood samples were collected during scanning to measure the metabolite-corrected input function as previously described (32). The plasma free fraction was measured in triplicate with ultrafiltration techniques as previously described (25).

MRI data were used to provide careful anatomic localization of ${ }^{11} \mathrm{C}$ PBR28 uptake in brain. MR data were acquired using a 3-T Siemens Trio scanner with a weighted gradient-echo sequence (echo time, 3.3 $\mathrm{ms}$; inversion time, $1,100 \mathrm{mS}$; repetition time, $2,500 \mathrm{~ms}$; fractional anisotropy, $7^{\circ}$ ) to result in images with $1 \mathrm{~mm}^{3}$ isotropic resolution.

\section{Imaging Data Analysis}

Dynamic list-mode emission data were histogrammed in time frames ranging from $30 \mathrm{~s}$ to $5 \mathrm{~min}$ and reconstructed with the motion-compensation ordered-subsets expectation maximization listmode algorithm for resolution recovery, which includes event-byevent motion correction. The first $10 \mathrm{~min}$ of PET data were summed and registered to the subject's anatomic MR data using a mutual information algorithm constrained to $6^{\circ}$ of freedom (Oxford Centre for Functional Magnetic Resonance Imaging of the Brain's Linear Image Registration Tool, Functional Magnetic Resonance Imaging of the Brain Software Library, version 3.2). The native MR image was coregistered into the Montreal Neurologic Institute template space using a nonlinear transformation algorithm (BioImage Suite). Atlas-defined bilateral regions of interest were transformed from the Automated Anatomic Labeling template space (33) to native PET space to extract ${ }^{11} \mathrm{C}-\mathrm{PBR} 28$ time-activity curves. Analyzed brain regions included the amygdala, hippocampus, caudate, putamen, thalamus, whole cerebellum, and frontal, occipital, parietal, and temporal cortices.

The primary outcome measure for all PET scans was ${ }^{11} \mathrm{C}-\mathrm{PBR} 28$ $V_{\mathrm{T}}$, the equilibrium ratio of ${ }^{11} \mathrm{C}-\mathrm{PBR} 28$ concentration in tissue to that in arterial plasma. Values of $V_{\mathrm{T}}$ were estimated with multilinear analysis $(34)\left(t^{*}=30 \mathrm{~min}\right)$ as previously validated, which yields a testretest reproducibility of $7 \%-9 \%$ in our center (32).

\section{Statistical Analysis}

Student $t$ tests were used to compare age, Beck Depression Inventory scores, and radiotracer injection parameters between smokers and nonsmokers. A linear mixed modeling approach was used for statistical analysis of ${ }^{11} \mathrm{C}-\mathrm{PBR} 28 V_{\mathrm{T}}$ values. To test the null hypothesis of no difference in ${ }^{11} \mathrm{C}$-PBR2 $8 V_{\mathrm{T}}$ between smokers and nonsmokers, a single model was constructed featuring $r$ r6971 genotype as a fixed factor, smoking status as a between-subjects factor, and brain region as a within-subject factor. Post hoc linear contrasts examined regional differences in ${ }^{11} \mathrm{C}$-PBR $28 V_{\mathrm{T}}$ between smokers and nonsmokers and were considered significant for an $\alpha$ value of less than 0.05 (uncorrected for multiple comparisons). As a secondary analysis, the same statistical approach was repeated using brain SUV (instead of $V_{\mathrm{T}}$ ), averaged 90-120 min after injection, as an outcome measure.

Exploratory analysis with linear regression examined for potential relationships between smoking behaviors (cigarette use, time of last cigarette, Fagerström Test for Nicotine Dependence, and Minnesota Nicotine Withdrawal Scale; smoking group only) or mood (Beck Depression Inventory; all subjects) with ${ }^{11} \mathrm{C}-\mathrm{PBR} 28 V_{\mathrm{T}}$ values. For these analyses, $r s 6971$ genotype was accounted for by creating a $z$ score for each variable within each genotype group (medium-affinity binder or high-affinity binder, not separated by smoking status for Beck Depression Inventory) as previously described (35). Linear regression analysis was performed on these variables with no correction for multiple comparisons because of the exploratory nature of the data.

\section{RESULTS}

On average, tobacco smokers $(n=20)$ reported smoking $15 \pm$ 8 cigarettes/d for $16 \pm 9 \mathrm{y}$, with Fagerström Test for Nicotine Dependence scores of $6 \pm 2$, Urge to Smoke Questionnaire-Intent scores of $10 \pm 4$, and Urge to Smoke Questionnaire-Relief scores of $11 \pm 5$. These characteristics are consistent with moderate to high levels of nicotine dependence (Table 1). There were no significant group differences in age or Beck Depression Inventory score. The injected PBR28 mass was greater in nonsmokers than in smokers $(P=0.002)$. There were no significant group differences in injected ${ }^{11} \mathrm{C}-\mathrm{PBR} 28$ radioactivity dose or plasma free fraction. 
TABLE 1

Subject and ${ }^{11} \mathrm{C}-\mathrm{PBR} 28$ Imaging Parameters

\begin{tabular}{|c|c|c|}
\hline Parameter & Nonsmokers $(n=20)$ & Smokers $(n=20)$ \\
\hline Age (y) & $31.0 \pm 12.2$ & $34.6 \pm 10.2$ \\
\hline Sex & $13 \mathrm{M} ; 7 \mathrm{~F}$ & $15 \mathrm{M} ; 5 \mathrm{~F}$ \\
\hline rs6971 genotype & $11 \mathrm{HAB} ; 9$ MAB & $15 \mathrm{HAB} ; 5 \mathrm{MAB}$ \\
\hline Cigarettes/d & NA & $15 \pm 8$ \\
\hline Fagerström Test for Nicotine Dependence & NA & $6 \pm 2$ \\
\hline \multicolumn{3}{|l|}{ Urge to Smoke Questionnaire } \\
\hline Intent & NA & $10 \pm 4$ \\
\hline Relief & NA & $11 \pm 5$ \\
\hline Beck Depression Inventory & $1 \pm 2$ & $3 \pm 3$ \\
\hline Injected dose (MBq) & $575 \pm 174$ & $505 \pm 188$ \\
\hline Injected mass $(\mu \mathrm{g})$ & $2.3 \pm 1.9$ & $0.79 \pm 0.60$ \\
\hline Plasma free fraction (\%) & $2.3 \pm 0.8$ & $2.6 \pm 0.9$ \\
\hline
\end{tabular}

Analysis of ${ }^{11} \mathrm{C}$-PBR28 $V_{\mathrm{T}}$ values did not reveal a significant main effect of smoking $\left(F_{1,36}=0.16, P=0.70\right.$; effect of genotype, $F_{1,36}=52.88, P<1^{\times} 10^{-8}$; smoking status-by-region interaction, $\left.F_{9,354}=1.8, P=0.07\right)$. Although these results indicated a trend for a smoking status-by-region interaction, post hoc linear contrasts revealed no significant differences in ${ }^{11} \mathrm{C}-\mathrm{PBR} 28 V_{\mathrm{T}}$ between smokers and nonsmokers in any region. The region with the largest group difference was thalamus $\left(F_{1,37}=0.69, P=0.41\right)$. To better characterize this null finding, we estimate a genotype-corrected whole-brain effect size (Cohen $d$ ) of 0.09 , with a $95 \%$ confidence interval of -0.44 to 0.62 . These findings are illustrated in Figure 1. For completeness, individual values are shown uncorrected for genotype in Supplemental Figure 1 (supplemental materials are available at http://jnm.snmjournals.org). No significant relationships between ${ }^{11} \mathrm{C}-\mathrm{PBR} 28 V_{\mathrm{T}}$ and mood or smoking measures were detected.

Secondary analyses of ${ }^{11} \mathrm{C}-\mathrm{PBR} 28$ SUVs similarly revealed no significant main effect of smoking $\left(F_{1,36}=0.22, P=0.64\right.$; effect of genotype, $\left.F_{1,32}=7.5, P=1 \times 10^{-3}\right)$ but a significant smoking status-by-region interaction $\left(F_{9,354}=2.45, P=0.01\right)$. Post hoc linear contrasts did not identify significant differences in ${ }^{11} \mathrm{C}$ PBR28 SUVs in any regions. The region with the largest group difference was thalamus $\left(F_{1,37}=0.91, P=0.35\right)$. These findings are illustrated in Supplemental Figure 2.

\section{DISCUSSION}

This study did not find evidence of differences in TSPO availability (measured by ${ }^{11} \mathrm{C}-\mathrm{PBR} 28 V_{\mathrm{T}}$ ) between tobacco smokers and nonsmokers, despite adequate power to detect moderate effect sizes. Since TSPO levels are sensitive to microglia activation, these findings suggest that microglia may not be chronically activated in tobacco smokers. Caution in this interpretation is required because TSPO levels may be affected by other factors, including altered TSPO expression in nonmicroglial cells, change in the numbers of cells expressing TSPO, or abnormal cell metabolism (36). Nonetheless, the result is not consistent with preclinical reports of increased neuronal cytokine expression and oxidative stress markers after tobacco smoke exposure $(20,21)$. Therefore, the neuroimmune effects of tobacco smoking are likely subtler than chronic activation of the brain immune system.

The present finding is not consistent with previous TSPO PET imaging reports that tobacco smokers exhibited lower brain SUV levels at early times after injection (20-40 $\mathrm{min}$ ) than nonsmokers $(22,23)$. Considering that our study exhibits sufficient power to detect medium effect sizes, underlying methodologic issues likely explain the seemingly conflicting results. An interpretation that synthesizes our data with these previous reports is that smokers have a different radiotracer distribution in the periphery or differences in radiotracer influx, whereas TSPO availability is comparable between the 2 groups. Differences in radiotracer metabolism or bioavailability are a well-known phenomenon in scenarios such as exogenous drug administration (37) or disease states (38). This same mechanism could explain the reported associations of brain SUV with number of cigarettes smoked per day found using a different radiotracer, ${ }^{11} \mathrm{C}$-DAA1106 (39). In our sample with the radiotracer ${ }^{11} \mathrm{C}$-PBR28, we did not find evidence of significant group differences in brain SUV at late times (90-120 min after injection). This finding may result from differences in the kinetic properties of the 2 radioligands used (40), low levels of specific binding with ${ }^{11} \mathrm{C}$-DAA1106, or the use of a later time frame (chosen to reduce the influence of radiotracer delivery and flow effects on SUVs). In summary, the fully quantitative analyses performed here do not indicate differences in TSPO availability between tobacco smokers and nonsmokers.

Studies of baseline TSPO availability do not definitively address the question of the possible immunosuppressive effects of tobacco smoking on the brain. A single scan does not capture the dynamic nature of the brain immune system. Approaches that measure the TSPO response to an acute immune stimulus such as endotoxin can provide an immune stress test reflecting the capacity of neuroimmune response (i.e., microglial activation) in study populations $(27,41)$. These studies are of high interest for future 


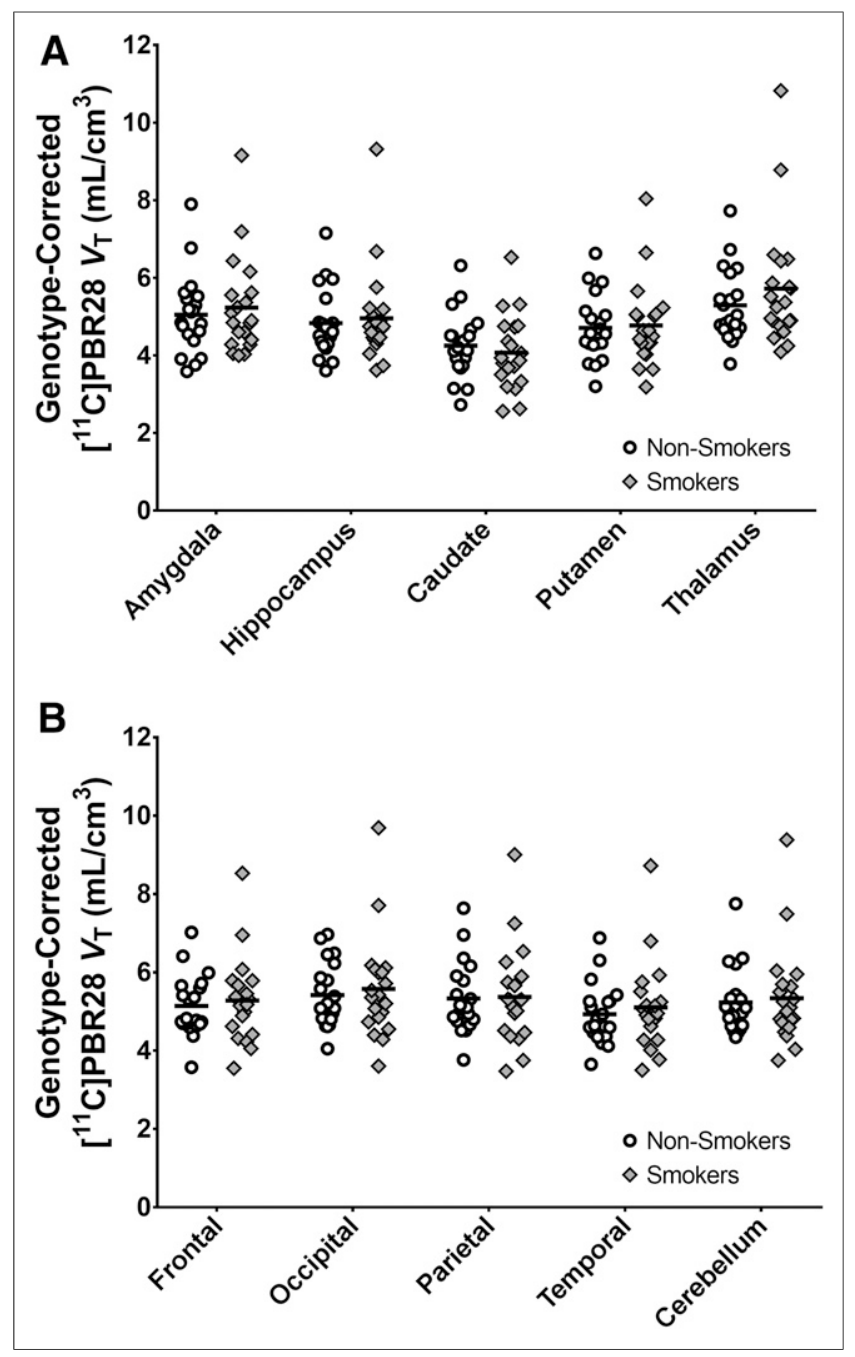

FIGURE 1. ${ }^{11} \mathrm{C}-\mathrm{PBR} 28 V_{\mathrm{T}}$ values do not significantly differ between smokers $(n=20)$ and nonsmokers $(n=20)$. ${ }^{11} \mathrm{C}$-PBR28 $V_{\mathrm{T}}$ values are adjusted for $r s 6971$ genotype for visualization purposes by incorporating genotype coefficient estimated by mixed model.

work to determine whether tobacco smoking suppresses (or exacerbates) neuroimmune response. Alternatively, newer imaging targets such as cyclooxygenase-2 (42) or reactive oxygen species (43) may provide biomarkers more directly related to tissue damage (i.e., inflammation). Such approaches may improve our understanding of the effects of tobacco smoking on brain immune signaling.

There are several limitations to this study. First, the injected PBR28 mass was significantly greater in nonsmokers than in smokers. A large injected mass can reduce outcome measures by occupying target sites with excess cold mass. However, the absolute injected mass levels were still quite low, and there was no evidence of a relationship between injected mass and ${ }^{11} \mathrm{C}-\mathrm{PBR} 28 V_{\mathrm{T}}$ in any region $\left(r^{2}<0.025 ; P>0.35\right)$. Therefore, mass effects likely did not influence these data. Second, given the low number of female smokers who participated in this study, it remains unknown whether male and female cigarette smokers have differences in TSPO availability. This question is particularly important in light of numerous sex differences in immune function (44) and is of high interest for future work. Because of this caveat, matching controls for subject sex and smoking status in future TSPO PET studies is still encouraged to account for these important factors (45). Third, the time of last nicotine exposure was not controlled in this study. Although no evidence of relationships between time of last cigarette and ${ }^{11} \mathrm{C}$ PBR28 $V_{\mathrm{T}}$ were observed, this study was not sufficiently powered to systematically assess such possible effects. Finally, the effects of menthol cigarettes on TSPO availability were not investigated (46). These issues are important questions for future research.

\section{CONCLUSION}

Using rigorous quantitative methods we find no evidence of significant differences in TSPO availability (i.e., ${ }^{11} \mathrm{C}-\mathrm{PBR} 28 V_{\mathrm{T}}$ ) between tobacco smokers and nonsmokers. Future work is needed to elucidate fully specific immune consequences of tobacco smoking on the human brain.

\section{DISCLOSURE}

This work was funded by NIH grants K01 AA024788, R01 MH110674, and R01 DA038832 and by the VA National Center for PTSD. No other potential conflict of interest relevant to this article was reported.

\section{ACKNOWLEDGMENTS}

We thank the staff at the Yale PET center for their expertise and support of radiochemistry and imaging. We are grateful to Jon Mikael Anderson and the Yale Translational Brain Imaging staff for their support of this work.

\section{KEY POINTS}

QUESTION: Do TSPO levels differ between tobacco smokers and healthy nonsmokers?

PERTINENT FINDINGS: In comparing ${ }^{11} \mathrm{C}$-PBR28 volumes of distribution (estimated by kinetic modeling with metabolite-corrected arterial input function) in 20 tobacco smokers and 20 nonsmokers, we found no evidence of significantly different TSPO levels between the 2 groups.

IMPLICATIONS FOR PATIENT CARE: The findings suggest that possible immune consequences of tobacco smoking on the human brain are subtle. It is important to account for radiotracer concentrations in plasma for TSPO quantification with PET imaging.

\section{REFERENCES}

1. Center for Disease Control and Prevention. Morbidity and Mortality Weekly Report. Washington, DC: U.S. Department of Health and Human Services; 2015

2. Sopori M. Effects of cigarette smoke on the immune system. Nat Rev Immunol. 2002;2:372-377.

3. Rom O, Avezov K, Aizenbud D, Reznick AZ. Cigarette smoking and inflammation revisited. Respir Physiol Neurobiol. 2013;187:5-10.

4. Bermudez EA, Rifai N, Buring JE, Manson JE, Ridker PM. Relation between markers of systemic vascular inflammation and smoking in women. Am J Cardiol. 2002;89:1117-1119.

5. Helmersson J, Larsson A, Vessby B, Basu S. Active smoking and a history of smoking are associated with enhanced prostaglandin F2 $\alpha$, interleukin- 6 and F2-isoprostane formation in elderly men. Atherosclerosis. 2005;181: 201-207. 
6. Perricone C, Versini M, Ben-Ami D, et al. Smoke and autoimmunity: the fire behind the disease. Autoimmun Rev. 2016;15:354-374.

7. Harricharan R, Abboussi O, Daniels WMU. Addiction: a dysregulation of satiety and inflammatory processes. Prog Brain Res. 2017;235:65-91.

8. Lacagnina MJ, Rivera PD, Bilbo SD. Glial and neuroimmune mechanisms as critical modulators of drug use and abuse. Neuropsychopharmacology. 2017;42:156-177.

9. York EM, Bernier LP, MacVicar BA. Microglial modulation of neuronal activity in the healthy brain. Dev Neurobiol. 2018;78:593-603.

10. Sofuoglu M, Waters AJ, Mooney M, O’Malley SS. Minocycline reduced craving for cigarettes but did not affect smoking or intravenous nicotine responses in humans. Pharmacol Biochem Behav. 2009;92:135-140.

11. Gao Z, Nissen JC, Ji K, Tsirka SE. The experimental autoimmune encephalomyelitis disease course is modulated by nicotine and other cigarette smoke components. PLoS One. 2014;9:e107979.

12. Schain M, Kreisl WC. Neuroinflammation in neurodegenerative disorders: a review. Curr Neurol Neurosci Rep. 2017;17:25.

13. Nimmerjahn A, Kirchhoff F, Helmchen F. Resting microglial cells are highly dynamic surveillants of brain parenchyma in vivo. Science. 2005;308:1314-1318.

14. Brown GC, Neher JJ. Microglial phagocytosis of live neurons. Nat Rev Neurosci. 2014;15:209-216.

15. Li Q, Barres BA. Microglia and macrophages in brain homeostasis and disease. Nat Rev Immunol. 2018;18:225-242.

16. Fujita M, Imaizumi M, Zoghbi SS, et al. Kinetic analysis in healthy humans of a novel positron emission tomography radioligand to image the peripheral benzodiazepine receptor, a potential biomarker for inflammation. Neuroimage. 2008;40:43-52.

17. Kreisl WC, Fujita M, Fujimura Y, et al. Comparison of [ $\left[{ }^{11} \mathrm{C}\right]-(\mathrm{R})-\mathrm{PK} 11195$ and $\left[{ }^{11} \mathrm{C}\right]$ PBR28, two radioligands for translocator protein $(18 \mathrm{kDa})$ in human and monkey: implications for positron emission tomographic imaging of this inflammation biomarker. Neuroimage. 2010;49:2924-2932.

18. Owen DR, Gunn RN, Rabiner EA, et al. Mixed-affinity binding in humans with 18-kDa translocator protein ligands. J Nucl Med. 2011;52:24-32.

19. Innis RB, Cunningham VJ, Delforge J, et al. Consensus nomenclature for in vivo imaging of reversibly binding radioligands. J Cereb Blood Flow Metab. 2007;27: 1533-1539.

20. Khanna A, Guo M, Mehra M, Royal W. Inflammation and oxidative stress induced by cigarette smoke in Lewis rat brains. J Neuroimmunol. 2013;254:69-75.

21. Royal W, Can A, Gould TD, et al. Cigarette smoke and nicotine effects on brain proinflammatory responses and behavioral and motor function in HIV-1 transgenic rats. J Neurovirol. 2018;24:246-253.

22. Brody AL, Gehlbach D, Garcia LY, et al. Effect of overnight smoking abstinence on a marker for microglial activation: a $\left[{ }^{11} \mathrm{C}\right]$ DAA1106 positron emission tomography study. Psychopharmacology (Berl). 2018;235:3525-3534.

23. Brody AL, Hubert R, Enoki R, et al. Effect of cigarette smoking on a marker for neuroinflammation: a [ $\left.{ }^{11} \mathrm{C}\right] \mathrm{DAA} 1106$ positron emission tomography study. $\mathrm{Neu}$ ropsychopharmacology. 2017;42:1630-1639.

24. Owen DR, Yeo AJ, Gunn RN, et al. An 18-kDa translocator protein (TSPO) polymorphism explains differences in binding affinity of the PET radioligand PBR28. J Cereb Blood Flow Metab. 2012;32:1-5.

25. Hannestad J, DellaGioia N, Gallezot J-D, et al. The neuroinflammation marker translocator protein is not elevated in individuals with mild-to-moderate depression: a [ $\left.{ }^{11} \mathrm{C}\right] \mathrm{PBR} 28$ PET study. Brain Behav Immun. 2013;33:131-138.

26. Hillmer AT, Sandiego CM, Hannestad J, et al. In vivo imaging of translocator protein, a marker of activated microglia, in alcohol dependence. Mol Psychiatry. 2017;22:1759-1766.
27. Sandiego CM, Gallezot JD, Pittman B, et al. Imaging robust microglial activation after lipopolysaccharide administration in humans with PET. Proc Natl Acad Sci USA. 2015;112:12468-12473.

28. Beck AT. An inventory for measuring depression. Arch Gen Psychiatry. 1961;4:561571.

29. Etter J-F, Duc TV, Perneger TV. Validity of the Fagerstrom test for nicotine dependence and of the Heaviness of Smoking Index among relatively light smokers. Addiction. 1999;94:269-281.

30. Hughes JR, Hatsukami D. Signs and symptoms of tobacco withdrawal. Arch Gen Psychiatry. 1986;43:289-294

31. Tiffany ST, Drobes DJ. The development and initial validation of a questionnaire on smoking urges. Br J Addict. 1991;86:1467-1476.

32. Park E, Gallezot J-D, Delgadillo A, et al. ${ }^{11} \mathrm{C}-\mathrm{PBR} 28$ imaging in multiple sclerosis patients and healthy controls: test-retest reproducibility and focal visualization of active white matter areas. Eur J Nucl Med Mol Imaging. 2015;42:1081-1092.

33. Tzourio-Mazoyer N, Landeau B, Papathanassiou D, Crivello F, Etard O, Delcroix N. Automated anatomical labeling of activations in SPM using a macroscopic anatomical parcellation of the MNI MRI single-subject brain. Neuroimage. 2002;15:273-289.

34. Ichise M, Toyama H, Innis RB, Carson RE. Strategies to improve neuroreceptor parameter estimation by linear regression analysis. J Cereb Blood Flow Metab. 2002;22:1271-1281.

35. Kreisl WC, Lyoo $\mathrm{CH}, \mathrm{McGwier} \mathrm{M}$, et al. In vivo radioligand binding to translocator protein correlates with severity of Alzheimer's disease. Brain. 2013;136:2228-2238.

36. Notter T, Coughlin JM, Sawa A, Meyer U. Reconceptualization of translocator protein as a biomarker of neuroinflammation in psychiatry. Mol Psychiatry. 2018;23:36-47.

37. Finnema SJ, Halldin C, Bang-Andersen B, Bundgaard C, Farde L. Serotonin transporter occupancy by escitalopram and citalopram in the non-human primate brain: a [ ${ }^{11}$ C]MADAM PET study. Psychopharmacology (Berl). 2015;232:4159-4167.

38. Naganawa M, Lim K, Nabulsi NB, et al. Evaluation of pancreatic VMAT2 binding with active and inactive enantiomers of $\left[{ }^{18} \mathrm{~F}\right] \mathrm{FP}-\mathrm{DTBZ}$ in healthy subjects and patients with type 1 diabetes. Mol Imaging Biol. 2018;20:835-845.

39. Venneti S, Wang G, Nguyen J, Wiley CA. The positron emission tomography ligand DAA1106 binds with high affinity to activated microglia in human neurological disorders. J Neuropathol Exp Neurol. 2008;67:1001-1010.

40. Cumming P, Burgher B, Patkar O, et al. Sifting through the surfeit of neuroinflammation tracers. J Cereb Blood Flow Metab. 2018;38:204-224.

41. Hannestad J, Gallezot JD, Schafbauer T, et al. Endotoxin-induced systemic inflammation activates microglia: $\left[{ }^{11} \mathrm{C}\right] \mathrm{PBR} 28$ positron emission tomography in nonhuman primates. Neuroimage. 2012;63:232-239.

42. Kim M-J, Shrestha SS, Cortes M, et al. Evaluation of two potent and selective PET radioligands to image COX-1 and COX-2 in rhesus monkeys. J Nucl Med. 2018;59:1907-1912.

43. Hou C, Hsieh C-J, Li S, et al. Development of a positron emission tomography radiotracer for imaging elevated levels of superoxide in neuroinflammation. ACS Chem Neurosci. 2018;9:578-586.

44. Klein SL, Flanagan KL. Sex differences in immune responses. Nat Rev Immunol. 2016;16:626-638.

45. Hillmer AT. The importance of drug and sex effects in psychiatric research. Biol Psychiatry. 2018;84:e71-e72.

46. Brody AL, Mukhin AG, La Charite J, et al. Up-regulation of nicotinic acetylcholine receptors in menthol cigarette smokers. Int $J$ Neuropsychopharmacol. 2013;16:957-966. 